\title{
Urothelial carcinoma of the bladder in a pediatric patient
}

\author{
Elke E. Mau, MD, FRCSC; Michael P. Leonard, MD, FRCSC \\ Department of Surgery, Division of Pediatric Urology, Children's Hospital of Eastern Ontario, University of Ottawa, Ottawa, ON, Canada
}

Cite as: Can Urol Assoc J 2016;10(7-8):E268-9. http://dx.doi.org/10.5489/cuaj.3412

Published online July 12, 2016

\section{Abstract}

Urothelial carcinoma of the bladder in children and adolescents is rare. The World Health Organization database has recorded approximately 80 patients under age 16 that have been diagnosed with papillary bladder tumour since $1968 .^{1}$ We are reporting on our case of urothelial carcinoma diagnosed in a 14-year-old male who presented with painless gross hematuria.

\section{Case report}

Our patient is a 14-year-old South Asian male, who immigrated to Canada from the Philippines in 2012. He had an isolated episode of painless, gross hematuria in July 2015, after which he presented to his primary care physician and renal and bladder ultrasound was ordered. Referral was also made to urology at that time.

The patient's medical history was significant for a remote history of pulmonary tuberculosis for which he received treatment, as well as a history of an intestinal parasite. The remainder of the history was non-contributory for typical risk factors of urothelial carcinoma, including no history of chemotherapy, radiation, or chemical exposure. He and his family denied history of tobacco usage and there was no family history of genitourinary malignancy.

Physical examination revealed a normal abdominal and genitourinary examination.

Ultrasound revealed an irregular lobulated polypoid lesion on the right postero- lateral bladder wall measuring $26 \times 29 \times 17 \mathrm{~mm}$ (Fig. 1). The kidneys were unremarkable.

The patient was taken to the operating room for a cystoscopy and transurethral resection of bladder tumour. Cystoscopy revealed a papillary lesion on the right posterolateral bladder wall just distal to the ureteric orifice, consistent with what was seen on imaging. Due to the patient's post-pubertal stage of genital development, the pediatric resectoscope could not reach the tumour. We gently dilated his urethra with sounds and used the $26 \mathrm{~F}$ resectoscope for resection. The tumour was completely resected and a sample was taken at the base.

Pathology revealed a low-grade papillary urothelial neoplasm with no definite invasion into the muscularis mucosae. Also reported was a large inverted component, the presence of small nests of urothelial cells in the stalk, and the strong staining for CK5 and CD44. Final pathology read papillary low-grade urothelial carcinoma without invasion (Ta).

\section{Discussion}

Though uncommon, there have been several consistent findings in regards to papillary bladder tumour occurrence in children. Tumours are predominately low-grade, more common in males at a 3:1 ratio, more common in White compared to Black patients, and are most often located on the trigone. ${ }^{2}$ They also seem to have a lower rate of recurrence than similarly staged tumours in the adult population, possibly due to field change being a less likely etiology in the absence of carcinogen exposure. ${ }^{3}$ As in our case, painless gross hematuria is the presenting symptom in $>90 \%$ of cases. There are no specific risk factors in the small number of cases reported. Associations reported in children include passive smoking, exposure to cyclophosphamide or muscle relaxants, as well as possible association with specific syndromes, such as Turner's syndrome. ${ }^{4}$

Diagnosis can occasionally be delayed in pediatric patients. Since gross hematuria in children so rarely indicates a malignancy, we wish to emphasize the importance of entertaining bladder tumour in the differential diagnosis. More commonly identified etiologies for gross hematuria in children include urinary tract infection (UTI), irritation of the meatus or perineum, and trauma. Other less common causes include nephrolithiasis, sickle cell disease/trait, coagulopathy, glomerular disease (including post-infectious glomerulonephritis and IgA nephropathy), malignancies (e.g., Wilms' tumour), and drug-induced hemorrhagic cystitis, such as is seen with cyclophosphamide. ${ }^{5}$ 


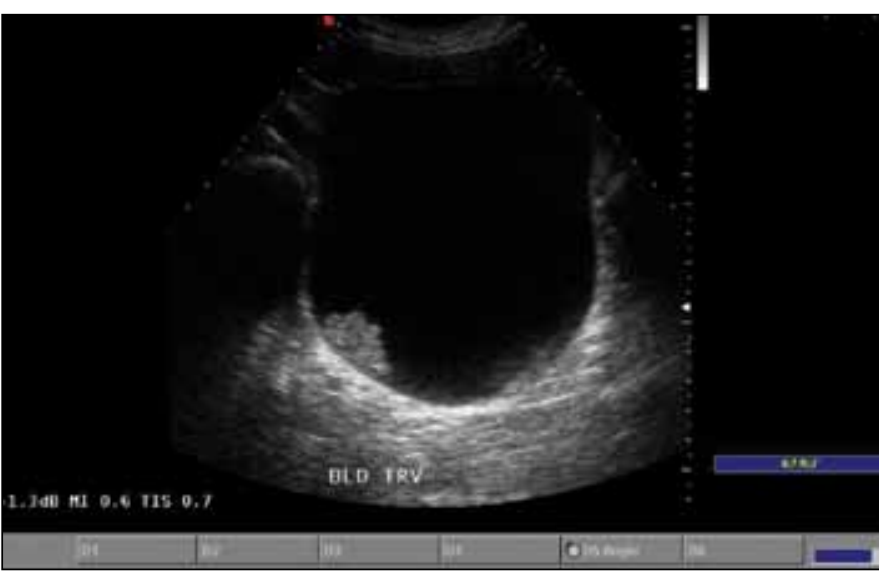

Fig. 1. Preoperative ultrasound showing patient's bladder wall mass.

Imaging of the urinary tract in a pediatric patient presenting with gross hematuria is indicated. Ultrasound is the imaging of choice in children to avoid radiation exposure and has been shown to have acceptable sensitivity rates regarding bladder tumour detection. ${ }^{2,5}$ Cytology may be ordered, but given the predominance of low-grade tumour in children, its usefulness may be limited. ${ }^{2}$

There are clear guidelines regarding the use of adjuvant intravesical immunotherapy and chemotherapy in adult patients; however, the use in children is not clear and the recommended treatment protocols may not be justified in light of the difference in clinical features and improved prognosis of urothelial carcinoma in children. Di Carlo et al summarized the papers published in the last 20 years and concluded omitting adjuvant treatment for low-grade urothelial carcinoma did not negatively affect the final outcome. ${ }^{6}$

Followup after initial resection is poorly defined in children. The need for general anesthetic for cystoscopy has led some to suggest considering ultrasound surveillance as an alternative. Hoenig et al found that ultrasound was $100 \%$ sensitive for detecting tumour and recommended ultrasound followup for low-grade tumours at one week post-operatively, then at three and six months, then annually. However, we would interpret this protocol with caution, given the very small series. ${ }^{3}$

The Canadian Urological Association (CUA) guidelines for adults would suggest followup cystoscopy for all patients at three months, followed by cystoscopy with cytology at 12 months, and then annually for Ta low-grade tumours.
Upper tract imaging is reserved for high-grade tumours. ${ }^{7}$ In the absence of clear recommendations for the pediatric patient, we will adhere to these guidelines for followup of the patient described above.

\section{Conclusion}

Urothelial carcinoma, though rare in the pediatric population, must be considered in the differential diagnosis of a child or adolescent presenting with gross hematuria. Ultrasound is indicated, followed in select cases by cystoscopy and transurethral resection to determine appropriate treatment and followup. Specific pediatric risk factors and appropriate followup regimens remain as yet poorly defined, and careful reporting of diagnosed cases is essential to elucidate these in the future.

Competing interests: The authors declare no competing personal or financial interests.

This paper has been peer-reviewed.

\section{References}

1. Fine SW, Humphrey PA, Dehner LP, et al. Urothelial neoplasms in patients 20 years or younger: A clinicopathological analysis using the World Health Organization 2004 bladder consensus classification. J Urol 2005;174;1976-80. http://dx.doi.org/10.1097/01.ju.0000176801.16827.82

2. Lerena J, Kravel L, Garcia-Aparicio L, et al. Transitional cell carcinoma of the bladder in children and adolescents: Six-case series and review of the literature. J Pediatr Urol 2010;6:481-5. http://dx.doi. org/10.1016/i.purol.2009.11.006

3. Hoenig DM, McRae S, Chen SC, et al. Transitional cell carcinoma of the bladder in the pediatric patient. J Urol 1996;156:203. http://dx.doi.org/10.1016/S0022-5347(01)66000-2

4. Aguiar L, Danialan R, Kim C. A case of high-grade transitional cell carcinoma of the bladder in a pediatric patient with Turner syndrome. Urology 2015;85:1477-9. http://dx.doi.org/10.1016/i.urology.2014.12.038

5. Greenfield SP, Williot P, Kaplan D. Gross hematuria in children: A 10-year review. Urology 2007;68:166. http://dx.doi.org/10.1016/i.urology.2006.10.018

6. Di Carlo D, Ferrari A, Perruccio K, et al. Management and followup of urothelial neoplasms of the bladder in children: A report from the TREP Project. Pediatr Blood Cancer 2015;62:1000-3.

7. Kassouf W, Traboulsi SL, Kulkarni GS, et al. CUA guidelines on the management of non-muscle invasive bladder cancer. Can Urol Assoc J 2015;9:E690-704. http://dx.doi.org/10.5489/cuai.3320

Correspondence: Dr. Michael P. Leonard, Department of Surgery, Division of Pediatric Urology, Children's Hospital of Eastern Ontario, University of Ottawa, Ottawa, ON, Canada; mleonard@cheo.on.ca 